\title{
The importance of co-design in the development of an e-Supportive Care System to support families with children and young people with complex health care needs
}

\author{
Dr Lisa McCann, Margaret Moore \\ School of Health Sciences \\ Faculty of Health \& Medical Sciences \\ University of Surrey \\ $6^{\text {th }}$ Floor, Standard Buildings, \\ 94 Hope Street, Glasgow \\ Scotland G2 6PH \\ I.mccann@surrey.ac.uk,m.moore@surrey.ac.uk
}

\begin{abstract}
An exploratory research project has been designed to inform the content and development of a prototype e-Supportive care system to support children and young people with undiagnosed complex health care needs and their families. A range of data collection activities with families and professionals have been conducted to ensure the system that is developed is informed by the experiences of potential users. This work is currently ongoing; the presentation will focus on the different recruitment strategies and data collection methods and the importance of co-design in the development of an e-Supportive care system.
\end{abstract}

Co-design. Complex health care needs. Families. Health Care Professionals.

\section{INTRODUCTION}

SWAN estimate that per annum in the UK, around 6,000 children with disabilities are born with an undiagnosed genetic condition [1].

Children and young people with no identified diagnosis may be described as having global developmental delay, experience learning and/or physical disabilities, they may have very complex and exceptional health care needs and can live with and experience a range of different symptoms [1].

Significant treatment developments and improvements mean increasing numbers of children and young people are living with long-term complex and continuing health care needs [2]. Providing coordinated and family-centred care in the home environment is vital [3] and central to health service provision for this population [4].

The family has a key role in providing and managing this care, which makes effective interactions and communications with professionals and specialist services essential to ensure optimal support for the family as a whole [4].
Therefore, the development of an e-Supportive care system is particularly important to meet people's ongoing and changing needs. However, to ensure that any eHealth system developed is reflective of people's needs, it is important to understand people's experiences of providing care to children and young people with complex healthcare needs to help inform its development and purpose.

\section{PURPOSE OF THE STUDY}

This Stage I exploratory research project has been designed to inform the content and development of a prototype e-Supportive care system to support children and young people with complex and exceptional health care needs and their families. There are four interlinked research objectives:

(i) Identify typical care pathways and understand the supportive care needs of children and young people who have no formal diagnosis for their complex and exceptional health care needs and their families. 
(ii) Understand the potential role of e-Health technologies to assist the supportive care needs of this population.

(iii) Develop, from these understandings, the content for a prototype e-Supportive care system.

(iv) Identify appropriate technologies and technological platforms to support the content and use of the e-Supportive care system in a future Stage II of this work.

\section{METHODS}

\section{Recruitment strategies}

Families and HCPs were recruited using a mixture of traditional strategies via recruiting clinicians and via more creative advertisements placed on websites, blogs, and social media sites of charity and family support organisations. Of the 39 families identified either through self or clinician referral, 15 families agreed to participate in an interview and 4 families agreed to participate in a survey. However, 1 family were lost prior to the interview taking place and 3 families did not return their survey. Therefore, a total of 14 families were interviewed and 1 family survey was returned.

The research team worked closely with parent representative members of the Project Steering Group to redesign the study's recruitment poster. The impact of this redesign was evident not only in the poster format and content itself, but also the improvements in study uptake following the redissemination of the revised recruitment poster.

\section{Data Collection}

There were a number of data collection strands in this research project, including:

(i) Mapping Survey with health care professionals in one NHS Board area in Scotland. Nine completed surveys were returned.

(ii) Focus groups/interviews and surveys with health care professionals (HCPs). Five focus groups, 1 interview and 11 surveys were completed. A multidisciplinary range of health care professionals are represented.

(iii) Surveys and interviews with families with children and young people with undiagnosed complex health care needs. Survey completion was low ( $n=1$ returned) but 14 families participated in individual interviews to share their experiences and share their ideas for their ideal e-Supportive care system.
The topic guides used in the semi-structured interviews with families and HCPs and in focus groups with HCPs focussed on the supportive care needs of children and young people with undiagnosed complex health care needs, the supportive care families receive at home and suggestions for the role of technology in the care of families, including barriers and facilitators to use.

\section{Member Checking}

An online forum was developed to share some of the findings and feedback from the study with family and HCP participants. The online forum was developed as a secure area, with username and password access required. Families and HCPs who participated in an interview or focus group were directly invited to join the eForum to provide any thoughts or further feedback on the suggested areas for development for the e-Supportive care system.

\section{Data Analysis}

Thematic Framework Analysis informed analysis of the qualitative data $[5,6]$. The researchers identified themes and developed a coding index which was used to code the transcripts using the NVivo analysis package. Data was sorted and reviewed, then charted thematically and synthesised into matrices. The matrices were reviewed and substantive content was identified.

\section{FINDINGS AND DISCUSSION}

The study has involved both HCPs and families, with ideas and experiences informing the understanding of the potential role of e-health technologies in the supportive care needs of children and young people with complex and exceptional healthcare needs.

Families and health care professionals have proposed a number of e-Supportive care solutions to help support families with children and young people with undiagnosed complex health care needs.

\section{Symptom management}

Families spoke about the complexities of the dayday symptom experiences of their children and young people and the impact these experiences have on the lives of all members of the family:

"And probably from about last August, she went from having these episodes of seizure like events which were originally like once a month or once every couple of months to every day and now she can have 10 to 15 a day. All day, every day, of episodes. Some last 30,40 seconds, some are lasting 10, 15 minutes and then she's up for 3 or 4 hours or more a night and she sits just having 
these episodes and seizures, whatever they are." (Mother, Family 11)

Similarly, HCPs recognised that the symptom profiles of children and young people with undiagnosed complex health care needs can be extremely variable. The need to, but difficulties of, developing a symptom management supportive care system reflective of the needs of different children and young people was discussed by HCPs:

"Well a lot of, we're talking about children that have, are undiagnosed, a lot of the children that we will see here have got a life, their life is expected to be shortened and they have, they've quite complex nursing and medical needs which can sometimes be similar to those children that do have a diagnosis ... and we're looking at things like respiratory and neurology, epilepsy, chest infections and $\mathrm{Gl}$ problems ... that type of thing." (F1, HCP Focus Group 3)

\section{Communication tool}

Many of the families spoke about the realities and challenges of receiving care for their child or young person from multiple specialties and multiple health care professionals. Many of the parents interviewed have a central administrative role in their child or young person's care. Not only is this extremely time consuming on a day-to-day basis for parents, it is also very frustrating. The challenges and limitations of the use of stand-alone systems between different professionals was noted by families and HCPs alike:

"And it's me that seems to be organising it all. And I think, that's just extra work that I don't think I should have to do. Whereas if everyone's connected into emails or whatever together, then everyone would know what was going on. Instead of me going, "Right. If you do this and l'll get him to do that and then l'll phone you back," and I'm constantly going, "Right, well if you do that, I'll do this and you do it." You know? Why are we not all connected together in one system so they all know what's going on with one child and...her whole things, you know?" (Mother, Family 11)

F1: I think a lot of the different are., areas, different hospitals, different departments,, they have different systems, like we've got our own system for notes, different NHS Boards seem to have different electronic note systems, GPs have a different electronic notes system and none of them actually link up ...
F2: $\quad \ldots$ so in terms of like transfer of information and things it's, it is difficult isn't it? [slight laugh]

F1: $\quad$ Aye, none o' them speak to each other.

$$
\text { (HCP Focus Group 2) }
$$

\section{Change current models of care delivery?}

It was evident from family narratives that current models of care delivery are not always fit for purpose, particularly as they often require considerable logistical planning and arrangements to accommodate a hospital appointment. For many families, the frustration surrounding these current arrangements are particularly acute when the value of some face-to-face appointments are limited. Therefore, families, and professionals, saw a key role of an e-Supportive Care System in terms of changing current models of care delivery, particularly in terms of changing the dynamic and arrangements for certain hospital appointments:

"But for 6 monthly appointment check-ups that you go and you say 'Oh we're all fine, no problems, but we've just sat in the car for an hour and then your waiting room for an hour and half. Now we're here 3 minutes and then we're going home again - thanks for that.' And l've had to pick ... saw other people picking my children up from school and all the rest of it. They don't see that, they see the 3 minutes you're sat there. They don't see the hours of organising to get there that it's taken and all of that. So that's a massive, massive function to drop that for a start." (Mother, Family 1 )

"The number of appointments some of these families have to go to, it becomes a huge burden doesn't it. Like some of them it can almost be like a full-time job ... coming to appointments..." (HCP Focus Group 2)

This project has embedded experiences-based user co-design in various stages of its design, development and conduct. It is evident that understanding the day-to-day experiences of families with children and young people with undiagnosed complex health care needs and various health care professionals who provide their care has been central to this study. Collectively, these experiences have informed a number of potential meaningful, experientially-base eSupportive care interventions. Future work will seek to develop and test a new system directly informed by the experiences of participants in this study. 


\section{ACKNOWLEDGEMENTS}

The research team would like to thank WellChild for funding this study. They would also like to thank all the families and Health Care Professionals who participated in the study and members of the Project Steering Group for their strategic input into the project.

\section{REFERENCES}

[1] SWAN UK. ABOUT SWAN UK. Available from: https://www.undiagnosed.org.uk/about-us/ (October 2016)

[2] Department of Health (2004) National Service Framework for Children, Young People and Maternity Services: Disabled children and young people and those with complex health care needs, London.
[3] Hewitt-Taylor, J, Farasat H. (2006) Learning to support children with complex needs in their homes. British Journal of Community Nursing, 11(5):209-13.

[4] Hunt, A., Coad, J., West, E. et al. (2012) The Big Study Report: Full findings report, Together for Short Lives, Bristol.

[5] Spencer L, Ritchie J, O'Connor W. (2008) Analysis: Practices, Principles and Processes. In: Jane Ritchie JL, ed. Qualitative Research Practice a Guide for Social Science Students and Researchers. London: SAGE Publications Ltd. 199-218.

[6] Ritchie J, Spencer L, O'Connor W. (2008) Carrying out Qualitative Analysis. In: Jane Ritchie JL, ed. Qualitative Research Practice a Guide for Social Science Students and Researchers. London: SAGE Publications Ltd 219-62. 